Wien klin Mag 2014 · 17:1

DOI 10.1007/s00740-014-0022-5

Online publiziert: 8. November 2014

(c) Springer-Verlag Wien 2014

Verena Kienast

Wien, Österreich

\title{
Dunkel ist das Leben ist der Tod
}

Die finanzielle Gesundung der österreichischen Krankenkassen hat naturgemäß Erwartungen und Hoffnungen auf eine Ausweitung mancher Leistungen für die Versicherten geweckt - vor allem, was die psychotherapeutische Therapie betrifft. Der neue Vorsitzende des Hauptverbands der österreichischen Sozialversicherungsträger, Peter McDonald, hat in seinen ersten Stellungnahmen derartige Hoffnungen - wohl ebenso naturgemäß - gleich gedämpft. Im Bereich der Zahnmedizin werde verhandelt, aber es sei auch zu bedenken, dass - erfreulicherweise - laufend neue hoch wirksame Therapien entwickelt werden, die hohe Kosten verursachen und den Handlungsspielraum dadurch einschränken. Die wirtschaftlich verantwortungsvolle Führung des Unternehmens Sozialversicherung ist fraglos notwendig, um die verlässliche Erfüllung der Aufgaben gewährleisten zu können. Allerdings wird es interessant sein, zu verfolgen, ob es irgendwann auch einmal eine volkswirtschaftliche Gesamtrechnung der Leistungen und dadurch erzielten Einsparungen in anderen Bereichen des Sozialsystems geben wird. Längerer Verbleib im Erwerbsleben, längerer Erhalt der Eigenständigkeit oder auch der Lebensqualität und damit der psychischen Gesundheit sind nur einige Beispiele, die in der Summe ein beträchtliches Maß an Einsparungen und - Stichwort: Steuerleistung auch an Einnahmen zur Folge haben.

\section{Nicht alles lässt sich messen}

Aber auch wenn es beispielsweise Instrumente zur Messung der Lebensqualität gibt, ist die Quantifizierung dieses komplexen Konstrukts schwierig und vor allem eher unzureichend in ein finanzielles Äquivalent umzusetzen. Zu unterschiedlich sind die persönlichen Prioritäten und Einschätzungen - ganz besonders in Hinblick auf den eigenen Tod. Wäh- rend manche Menschen mit einer tödlichen Krankheit versuchen, ihr bisheriges Leben so lange wie möglich aufrecht zu erhalten, möchten andere ihre letzte Möglichkeit nützen, jene Dinge zu tun, die sie immer schon tun wollten und immer verschoben haben, wie beispielsweise Reisen oder Freunde zu sehen, und andere ziehen sich lieber zurück und versuchen Ordnung in ihre Dinge zu bringen. In gewisser Weise wird der Tod von den meisten Menschen immer noch als Niederlage empfunden - und damit als Tabu betrachtet.

\section{Der Genuss des Augenblicks}

„Dunkel ist das Leben ist der Tod“ lautet die wiederkehrende Schlusszeile des „Trinklieds vom Jammer der Erde“ im „Lied von der Erde“ von Gustav Mahler, dessen Text auf chinesischen Gedichten basiert. Die fernöstliche Grundlage zeigt daher auch eine durchaus tröstliche Verbindung zwischen Leben und Tod. Der Genuss des Augenblicks gewinnt da große Bedeutung: JETZT leben wir, auch wenn das Leben im nächsten Moment vielleicht schon zu Ende ist. Es ist dieses JETZT das zählt:

Ein voller Becher Weins zur rechten Zeit Ist mehr wert, als alle Reiche dieser Erde!

Und dennoch:

\section{Dunkel ist das Leben, ist der Tod!}

Die westlichen Gesellschaften tendieren jedoch nach wie vor dazu, den Tod zu verdrängen und der medizinische Fortschritt drängt ihn auch beharrlich immer weiter zurück. Dem stehen Naturkatastrophen und kriegerische Auseinandersetzungen gegenüber, die über die weltweit in Sekundenschnelle zugänglichen Medien Bilder vom Massensterben zum
Bestandteil unseres Alltags machen. Eine höchst zwiespältige Situation, die uns die menschlichen Schwächen drastisch vor Augen führt, die wir gerne vor allzu viel Leid verschließen.

Die Fähigkeit zu leiden ist aber eng mit der Fähigkeit zur Freude verbunden, so wie das volle Glücksempfinden auch den Schmerz miteinschließt. Das sollte in den Kleinlichkeiten des Alltags nicht verloren gehen: Der Sinn für das Ganze

Meint Ihre

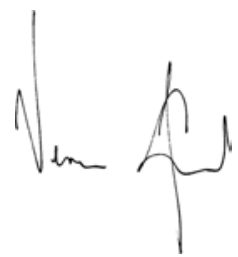

\section{Korrespondenzadresse}

\section{Kienast}

Wien

verena.kienast@springer.at 